\title{
Strange stars in $f(R)$ theories of gravity in the Palatini formalism
}

\author{
Grigoris Panotopoulos* \\ CENTRA, Instituto Superior Técnico, \\ Universidade de Lisboa, Av. Rovisco País 1, Lisboa, Portugal
}

(Dated: September 20, 2018)

\begin{abstract}
In the present work we study strange stars in $f(R)$ theories of gravity in the Palatini formalism. We consider two concrete well-known cases, namely the $R+R^{2} /\left(6 M^{2}\right)$ model as well as the $R-\mu^{4} / R$ model for two different values of the mass parameter $M$ or $\mu$. We integrate the modified TolmanOppenheimer-Volkoff equations numerically, and we show the mass-radius diagram for each model separately. The standard case corresponding to the General Relativity is also shown in the same figure for comparison. Our numerical results show that the interior solution can be vastly different depending on the model and/or the value of the parameter of each model. In addition, our findings imply that i) for the cosmologically interesting values of the mass scales $M, \mu$ the effect of modified gravity on strange stars is negligible, while ii) for the values predicting an observable effect, the modified gravity models discussed here would be ruled out by their cosmological effects.

PACS numbers: 04.40.Dg, 04.50.Kd
\end{abstract}

*Electronic address: grigorios.panotopoulos@tecnico.ulisboa.pt 


\section{INTRODUCTION}

Current astrophysical and cosmological observational data indicate that the Universe is expanding at an accelerating rate. The concordance model based on cold dark matter and a cosmological constant is the most economical model in excellent agreement with the data. However, the problems with the cosmological constant have given birth to many alternatives, based either on a new dynamical field or on modifications to Einstein's General Relativity (GR), see e.g. [1]. Of special interest are the models of $f(R)$ theories of gravity [2], which are a natural generalization of General Relativity and have been extensively studied as alternatives to the cosmological constant to describe the current cosmic acceleration.

Compact objects [3], such as white dwarfs and neutron stars, are the final fate of stars. The degeneracy pressure provided by the Fermi gas balances the gravitational force, and the star finds a stable configuration. In white dwarfs the Fermi gas consists of electrons, while in neutron stars the required pressure is provided by neutrons. Recently a new class of compact objects has been postulated to exist due to some observed super-luminous supernovae [4], which occur in about one out of every 1000 supernovae explosions, and are more than 100 times brighter than normal supernovae. One plausible explanation is that neutrons are further compressed so that a new object made of de-confined quarks is formed. This new compact object is called a "strange star", and since it is a much more stable configuration compared to a neutron star it could explain the origin of the huge amount of energy released in super-luminous supernovae [5]. Compact objects, due to their unique properties, comprise an excellent natural laboratory to study and perhaps constrain different models of modifications of gravity.

In the present work we wish to study strange stars in $f(R)$ theories of gravity. It is well known [2] that in studying $f(R)$ theories of gravity there are two different formalisms, namely the metric formalism and the Palatini formalism. In General Relativity they are completely equivalent, however in modified theories of gravity they lead to different predictions. It is known that $f(R)$ theories of gravity have difficulties with passing Solar System tests [6], and also with supporting interior star solutions [7]. In our work we have chosen to work in the Palatini formalism, since in this formalism the models are, on the one hand, easily compatible with Solar System tests, and on the other hand the interior star solution can match the exterior solution [8, 9]. See, however, [10] for evidence against the cosmological 
and astrophysical viability of the Palatini- $f(R)$ gravity.

We organize our work as follows: After this introduction, we present the theoretical framework in the next section. In the third section we discuss two specific models and we present in figures our numerical results. Finally we conclude in the last section.

\section{STRANGE STARS IN MODIFIED THEORIES OF GRAVITY}

\section{A. Relativistic stars in General Relativity}

In this subsection we review relativistic stars, and in particular strange stars, in GR. The starting point is Einstein's field equations with a cosmological constant

$$
G_{\mu \nu}+\Lambda g_{\mu \nu}=8 \pi T_{\mu \nu}
$$

where we have set Newton's constant equal to unity, $G=1$, and in the exterior problem the matter energy-momentum tensor vanishes. For matter we assume a perfect fluid with pressure $p$, energy density $\rho$ and an equation of state $p(\rho)$. The energy-momentum trace is given by $T=-\rho+3 p$. For the case of strange stars we shall consider the simplest equation of state corresponding to a relativistic gas of de-confined quarks, known also as the MIT bag model [11]

$$
p=\frac{1}{3}(\rho-4 B)
$$

and the bag constant has been taken to be $B=57 \mathrm{MeV} / \mathrm{fm}^{-3}$ (which is slightly smaller than the $59 \mathrm{MeV} / \mathrm{fm}^{3}$ required so that neutrons do not coagulate into droplets of ud matter in the simple MIT bag model, see the book by Haensel, Potekhin, Yakovlev, 2007) [12]). We mention in passing that for refinements of the bag model the reader could consult e.g. [13], and for the present state-of-the-art the recent paper [14]. For the metric in the case of static spherically symmetric spacetimes we consider the following ansatz

$$
d s^{2}=-f(r) d t^{2}+g(r) d r^{2}+r^{2} d \Omega^{2}
$$

with two unknown functions of the radial distance $f(r), g(r)$. For the exterior problem one obtains the well-known solution

$$
f(r)=g(r)^{-1}=1-\frac{2 M}{r}-\frac{\Lambda r^{2}}{3}
$$


where $M$ is the mass of the star. For the interior solution we introduce the function $m(r)$ instead of the function $g(r)$ defined as follows

$$
g(r)^{-1}=1-\frac{2 m(r)}{r}-\frac{\Lambda r^{2}}{3}
$$

so that upon matching the two solutions at the surface of the star we obtain $m(R)=M$, where $R$ is the radius of the star. The famous Tolman-Oppenheimer-Volkoff equations [15] for the interior solution of a relativistic star with a non-vanishing cosmological constant read [16]

$$
\begin{aligned}
m^{\prime}(r) & =4 \pi r^{2} \rho(r) \\
p^{\prime}(r) & =-(p+\rho) \frac{m(r)+4 \pi p r^{3}-\frac{\Lambda r^{3}}{3}}{r^{2}\left(1-\frac{2 m(r)}{r}-\frac{\Lambda r^{2}}{3}\right)}
\end{aligned}
$$

where the prime denotes differentiation with respect to $r$, and the equations are to be integrated with the initial conditions $m(r=0)=0$ and $p(r=0)=p_{c}$, where $p_{c}$ is the

central pressure. The radius of the star is determined requiring that the pressure vanishes at the surface, $p(R)=0$, and the mass of the star is then given by $M=m(R)$. Strange stars with the above equations of state in GR have been investigated in [16] for negative and positive values of the cosmological constant. The observed value of the cosmological constant is too small to have an effect. Only when $\Lambda$ is comparable to the bag constant B has an observable effect.

\section{B. Relativistic stars in $f(R)$ in the Palatini formalism}

We shall consider models described by an action of the form

$$
S=\int d^{4} x \sqrt{-g}\left(\frac{f(R)}{8 \pi}+\mathcal{L}_{M}\right)
$$

where $f(R)$ is a generic function of the Ricci scalar, and $\mathcal{L}_{M}$ is the matter Lagrangian density. In the Palatini formalism the field equations take the form [8]

$$
F(R) R_{\mu \nu}-\frac{1}{2} f(R) g_{\mu \nu}=8 \pi T_{\mu \nu}
$$

where $F(R)=d f(R) / d R$ is the first derivative of $f(R)$ with respect to $R$. Note that when $f(R)=R$ and $F(R)=1$ the field equations are reduced to the standard equations of GR. Taking the trace of the above field equations we obtain an algebraic equation relating the 
Ricci scalar $R$ to the trace of the matter energy-momentum tensor $T=-\rho+3 p$ as follows [8]

$$
R F(R)-2 f(R)=8 \pi T
$$

In cases where the trace $\mathrm{T}$ does not depend on $\mathrm{r}$, the field equations take the familiar form corresponding to Einstein's equations with a non-vanishing cosmological constant, namely [8]

$$
G_{\mu \nu}+\Lambda_{\rho} g_{\mu \nu}=\frac{8 \pi T_{\mu \nu}}{F_{\rho}}
$$

where the effective cosmological constant is given by

$$
\Lambda_{\rho}=\frac{1}{2}\left(R-\frac{f(R)}{F(R)}\right)
$$

which is evaluated at the value $R_{0}$ that solves the trace equation. The same holds for $F_{\rho}$. For a generic equation of state where the trace $\mathrm{T}$ is a function of the radial distance $\mathrm{r}$, one must integrate the full (more complicated) field equations as was done in [9] for polytropic stars. If we set $f(r)=e^{A(r)}$ and $g(r)=e^{B(r)}$, then the differential equations for the functions $A(r), B(r)$ are the following [8, 9]:

$$
\begin{aligned}
A^{\prime} & =-\frac{1}{1+\gamma}\left(\frac{1-e^{B}}{r}+\frac{\alpha}{r}-8 \pi r p \frac{e^{B}}{F}\right) \\
B^{\prime} & =\frac{1}{1+\gamma}\left(\frac{1-e^{B}}{r}+\frac{\alpha+\beta}{r}+8 \pi r \rho \frac{e^{B}}{F}\right)
\end{aligned}
$$

where $\gamma=\left(r F^{\prime}\right) /(2 F)$, while the $\alpha, \beta$ are given by

$$
\begin{aligned}
& \alpha=r^{2}\left(\frac{3}{4}\left(\frac{F^{\prime}}{F}\right)^{2}+\frac{2 F^{\prime}}{r F}+\frac{e^{B}}{2}\left(R-\frac{f}{F}\right)\right) \\
& \beta=r^{2}\left(\frac{F^{\prime \prime}}{F}-\frac{3}{2}\left(\frac{F^{\prime}}{F}\right)^{2}\right)
\end{aligned}
$$

Returning to the equation of state $p=(1 / 3)(\rho-4 B)$, for the exterior problem the energymomentum vanishes, while the effective cosmological constant becomes $\Lambda_{0}=\Lambda_{\rho}(T=0)$. Therefore, in the Palatini formalism for a constant energy-momentum trace the problem is reduced to the usual problem of GR with a non-vanishing cosmological constant, and with new pressure and energy density for the matter fluid $\tilde{p}=p / F_{\rho}$ and $\tilde{\rho}=\rho / F_{\rho}$. The modified pressure and energy density satisfy the same equation of state, but now the bag constant is modified by the same factor, namely $\tilde{B}=B / F_{\rho}$. Therefore we have to integrate the TOV 
equations valid in GR with the substitution $B \rightarrow \tilde{B}$. Finally, one more difference compared to GR comes from matching the two solutions (interior and exterior problems), since in $f(R)$ theories of gravity there are two different cosmological constants outside the star and inside the star. This implies that the mass of the star is now given by [9]

$$
M=m(R)+\frac{\left(\Lambda_{\rho}-\Lambda_{0}\right) R^{3}}{6}
$$

\section{NUMERICAL RESULTS IN TWO CONCRETE MODELS}

Here we shall consider two specific models, namely the Starobinsky model [17], $f(R)=$ $R+R^{2} /\left(6 M^{2}\right)$ as well as the $1 / R$ model that has been used to describe the current cosmic acceleration [18], $R-\mu^{4} / R$. Each model is characterized by a single parameter which is a mass scale. In Starobinsky's model the corrections to GR are important in the early universe, and we can have a successful inflationary model if $M \sim 10^{12} \mathrm{GeV}$, while in the second model the corrections are important at late times, and the mass scale $\mu \sim H_{0} \sim 10^{-33} \mathrm{eV}$ [18], where $H_{0}$ is today's Hubble parameter. In the cosmologically interesting range of the parameters $M, \mu$ the effect on the strange star is negligible. In this work, however, the mass scales are just two free parameters and we shall not consider the aforementioned values. Strange stars in $R^{2}$ gravity have also been studied in [19, 20], but in the Einstein frame, while in this article we work in the Jordan frame and in the Palatini formalism.

a) Starobinsky model: For a model of the form $f(R)=R+R^{2} /\left(6 M^{2}\right)$ the functions we need are found to be

$$
\begin{aligned}
R_{0} & =32 \pi B \\
F_{\rho} & =1+\frac{32 \pi B}{3 M^{2}} \\
\Lambda_{\rho} & =\frac{256 \pi^{2} B^{2}}{32 \pi B+3 M^{2}} \\
\Lambda_{0} & =0
\end{aligned}
$$

since for strange stars the energy-momentum trace is $T=-4 B$. We have considered two different values of $M$ for which $F_{\rho}=3 / 2$ and $F_{\rho}=2$. In Fig. 1 below we show the mass-

radius diagram for Starobinsky's model for $M=\sqrt{\frac{64 \pi B}{3}}$ and for $M=\sqrt{\frac{32 \pi B}{3}}$, and for comparison we also show the standard results corresponding to GR. 
b) $1 / R$ model: In this case the relevant functions are computed to be

$$
\begin{aligned}
& R_{0}=16 \pi B+\sqrt{3 \mu^{4}+256 \pi^{2} B^{2}} \\
& F_{\rho}=1+\frac{\mu^{4}}{\left(16 \pi B+\sqrt{256 \pi^{2} B^{2}+3 \mu^{4}}\right)^{2}} \\
& \Lambda_{\rho}=\frac{\mu^{4}\left(16 \pi B+\sqrt{256 \pi^{2} B^{2}+3 \mu^{4}}\right)}{\mu^{4}+\left(16 \pi B+\sqrt{256 \pi^{2} B^{2}+3 \mu^{4}}\right)^{2}} \\
& \Lambda_{0}=\frac{\sqrt{3} \mu^{2}}{4}
\end{aligned}
$$

We have considered two different values for the mass scale $\mu$ for which $F_{\rho}=1.05511$ and $F_{\rho}=1.11112$. In Fig. 2 below we show the mass-radius diagram for the two values $\mu=3 \sqrt{\pi B}$ and $\mu=4 \sqrt{\pi B}$ together with the GR results for comparison. The numerical results shown in Figures 1 and 2 clearly show that the interior solution of strange stars can be vastly different depending on the model and/or the value of the parameter of each model.

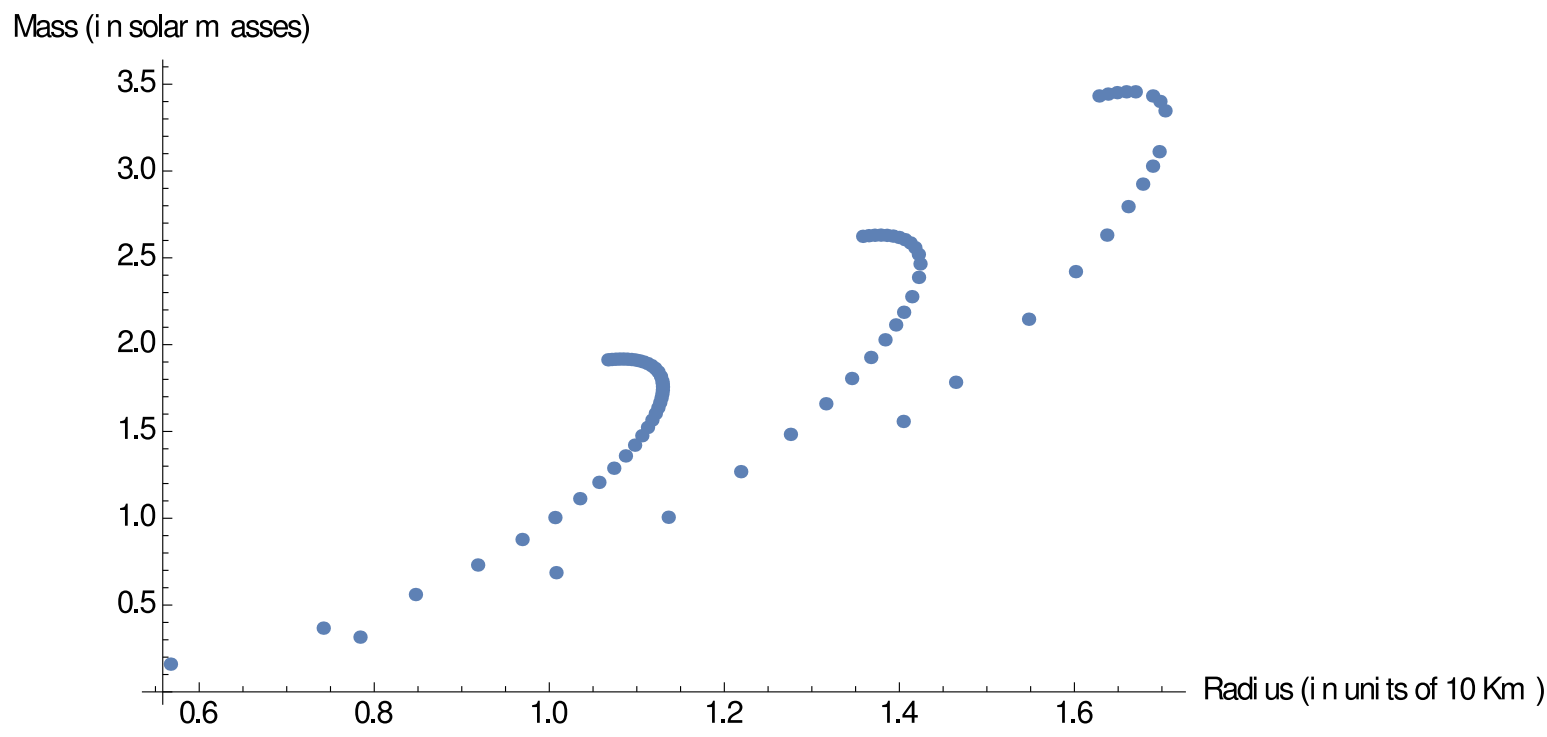

FIG. 1: Mass-radius diagram for quark stars in GR and in the Starobinsky model for two different values of the mass scale M. From left to right $F=1$ (GR), $F_{\rho}=3 / 2, F_{\rho}=2$.

A final comment is in order before finishing. Following [16, 19, 20] we have considered here the simplest equation of state corresponding to the bag model, namely radiation plus a bag constant. If a refined equation of state is used [13] the trace of the energy-momentum tensor, as stated before, will not be a constant any more, and the full field equations must be integrated. In this case one expects that the predictions of the same $f(R)$ models will 


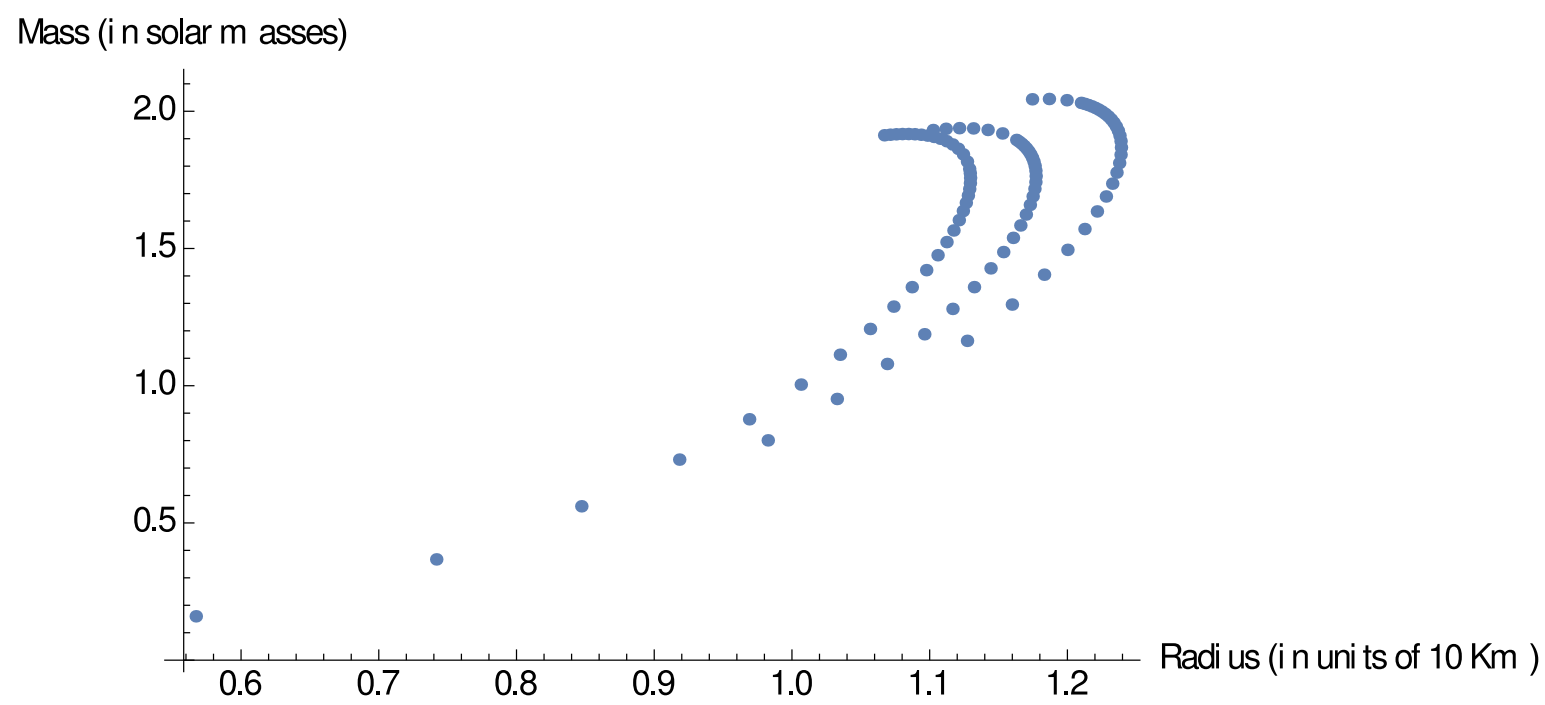

FIG. 2: Same as in Fig. 1 but for the model $R-\mu^{4} / R$. From left to right $F=1(\mathrm{GR}), F_{\rho}=1.05511$, $F_{\rho}=1.11112$.

change. It would be interested to see exactly how different the new predictions will be compared to the results obtained here, and we wish to be able to address that issue in a future work.

\section{CONCLUSIONS}

To summarize, in the present work we have studied strange stars in $f(R)$ theories of gravity in the Palatini formalism. On the one hand it is known that this class of modified theories of gravity can explain the current cosmic acceleration without a cosmological constant and also without introducing a new dynamical field. On the other hand strange stars are hypothetical compact objects, like neutron stars, which are made out of de-confined quarks. This composition in theory leads to a more stable configuration, and thus could explain the huge amount of energy released in the observed super-luminous supernovae, which are more than 100 times brighter than normal supernovae. We have considered the simple equation of state $p=(1 / 3)(\rho-4 B)$ from the MIT bag model, which in the framework of Einstein's General Relativity has already been investigated. Here we have considered two concrete well-known $f(R)$ models, namely the $R+R^{2} /\left(6 M^{2}\right)$ model as well as the $R-\mu^{4} / R$ for two different values of the mass parameter $M$ or $\mu$. We have integrated the modified Tolman-Oppenheimer-Volkoff equations numerically for many different values of the central 
pressure as an initial condition, and finally we have shown the mass-radius diagram for each case separately for two different values of the mass parameter $M$ or $\mu$. For comparison the standard diagram corresponding to General Relativity is also shown in the same figure. Our numerical results show that the interior solution can be vastly different depending on the model and/or the value of the parameter of each model. In addition, our findings imply that i) for the cosmologically interesting values of the mass scales $M, \mu$ the effect of modified gravity on strange stars is negligible, while ii) for the values predicting an observable effect, the modified gravity models discussed here would be ruled out by their cosmological effects.

\section{Acknowledgments}

The author wishes to thank the reviewers for valuable comments and suggestions. His work was support from "Fundação para a Ciência e Tecnologia".

[1] E. J. Copeland, M. Sami and S. Tsujikawa, Int. J. Mod. Phys. D 15 (2006) 1753 hep-th/0603057.

[2] S. Capozziello and M. Francaviglia, Gen. Rel. Grav. 40 (2008) 357 arXiv:0706.1146 [astro$\mathrm{ph}]] ;$

T. P. Sotiriou and V. Faraoni, Rev. Mod. Phys. 82 (2010) 451 [arXiv:0805.1726 [gr-qc]];

A. De Felice and S. Tsujikawa, Living Rev. Rel. 13 (2010) 3 arXiv:1002.4928 [gr-qc]].

[3] S. L. Shapiro and S. A. Teukolsky, "Black holes, white dwarfs, and neutron stars: The physics of compact objects," New York, USA: Wiley (1983) 645 p.

[4] E. O. Ofek et al., Astrophys. J. 659 (2007) L13 [astro-ph/0612408.

[5] D. Leahy and R. Ouyed, Mon. Not. Roy. Astron. Soc. 387 (2008) 1193 [arXiv:0708.1787 [astro$\mathrm{ph}]$;

R. Ouyed, D. Leahy and P. Jaikumar, arXiv:0911.5424 [astro-ph.HE];

D. Leahy and R. Ouyed, arXiv:1303.2047 [astro-ph.HE].

[6] X. H. Jin, D. J. Liu and X. Z. Li, astro-ph/0610854.

[7] T. Kobayashi and K. i. Maeda, Phys. Rev. D 78 (2008) 064019 [arXiv:0807.2503 [astro-ph]];

E. Babichev and D. Langlois, Phys. Rev. D 80 (2009) 121501 Erratum: [Phys. Rev. D 81 
(2010) 069901] [arXiv:0904.1382 [gr-qc]].

[8] K. Kainulainen, V. Reijonen and D. Sunhede, Phys. Rev. D 76 (2007) 043503 [gr-qc/0611132].

[9] K. Kainulainen, J. Piilonen, V. Reijonen and D. Sunhede, Phys. Rev. D 76 (2007) 024020 arXiv:0704.2729 [gr-qc]].

[10] T. Koivisto, Phys. Rev. D 73 (2006) 083517 astro-ph/0602031];

E. Barausse, T. P. Sotiriou and J. C. Miller, Class. Quant. Grav. 25 (2008) 105008 arXiv:0712.1141 [gr-qc]].

[11] Chodos A, Jaffe R L, Johnson K, Thorn C B, and Weisskopf V F, 1974a Phys. Rev. D. 9 3471-95;

Chodos A, Jaffe R L, Johnson K, and Thorn C B, 1974b Phys. Rev. D. 10 2599-2604;

Farhi E and Jaffe R L 1984 Phys. Rev. D. 302379.

[12] P. Haensel, A. Y. Potekhin and D. G. Yakovlev, "Neutron stars 1: Equation of state and structure," Astrophys. Space Sci. Libr. 326 (2007).

[13] V. D. Toneev, E. G. Nikonov, B. Friman, W. Norenberg and K. Redlich, Eur. Phys. J. C 32 (2003) 399 hep-ph/0308088;

Y. B. Ivanov, A. S. Khvorostukhin, E. E. Kolomeitsev, V. V. Skokov, V. D. Toneev and D. N. Voskresensky, Phys. Rev. C 72 (2005) 025804 [astro-ph/0501254].

[14] A. Vuorinen, arXiv:1611.04557 [hep-ph].

[15] J. R. Oppenheimer and G. M. Volkoff, Phys. Rev. 55 (1939) 374;

Tolman R C 1939 Phys. Rev. 55 364-73.

[16] O. Zubairi, A. Romero and F. Weber, J. Phys. Conf. Ser. 615 (2015) no.1, 012003.

[17] A. A. Starobinsky, Phys. Lett. B 91 (1980) 99.

[18] S. M. Carroll, V. Duvvuri, M. Trodden and M. S. Turner, Phys. Rev. D 70 (2004) 043528 astro-ph/0306438.

[19] K. V. Staykov, D. D. Doneva, S. S. Yazadjiev and K. D. Kokkotas, JCAP 1410 (2014) no.10, 006 arXiv:1407.2180 [gr-qc]].

[20] A. V. Astashenok, Int. J. Mod. Phys. Conf. Ser. $41 \quad$ (2016) 1660130. doi:10.1142/S2010194516601307 